\section{La Biblioteca Virtual en Equidad, Salud y Desarrollo Humano}

América Valdés ${ }^{1}$

Palabras clave: equidad en salud, BIREME, desarrollo.

\footnotetext{
1 Coordinación de Investigación, Organización Panamericana de la Salud, Washington, D.C., Estados Unidos de América. Dirección postal: 525 Twenty-third Street NW, Washington, DC 20037. Estados Unidos de América. Dirección electrónica: valdesam@paho.org

2 OPS. Documento Oficial de la OPS No. 296, junio de 2001.

3 Si desea mayor información puede visitar el sitio de Internet http:// www.bireme.br/bvc/E/documentosBVS.htm
}

La Biblioteca Virtual en Salud (BVS) es resultado y herencia de una labor conjunta, realizada durante más de tres décadas, para ampliar y fortalecer el flujo de información cientificotécnica en materia de salud en la Región de las Américas. Esta labor se efectuó bajo la dirección de la Organización Panamericana de la Salud (OPS) por conducto del Centro Latinoamericano y del Caribe para Información en Ciencias de la Salud (BIREME). La BVS ha de entenderse como una colección dinámica de fuentes de información que busca facilitar el acceso equitativo a la información y a los conocimientos en materia de salud. Desde sus orígenes, este trabajo conjunto se ha renovado continuamente de acuerdo con nuevos modelos para la gestión, organización y manejo de la información.

En 1998, la OPS propuso la BVS como una nueva estrategia de cooperación en información cientificotécnica adaptada a la actual era de Internet. Por este motivo se ha venido desarrollando como un nuevo paradigma en el uso y transmisión de información científica y técnica destinada a ayudar a los países a superar problemas estructurales de coordinación en el desarrollo, el acceso y la gestión de sistemas de información en la Región. ${ }^{2}$

La OPS ha planteado el desarrollo de la BVS en el ámbito temático de la equidad, la salud y el desarrollo humano con el fin de fomentar el uso de la información para la toma de decisiones en el ámbito de la salud y en las actividades científicas relacionadas con las políticas públicas, brindando acceso a información, datos, indicadores, documentos de política, metodología e instrumentos relacionados con este proceso. ${ }^{3}$ En este contexto, las "políticas públicas" son acciones organizadas en torno a objetivos de interés colectivo y, por lo tanto, no son un sinónimo de las políticas estatales.

Como sucede en otros aspectos de la salud, la toma de decisiones concernientes al mejoramiento de la equidad en el ámbito sanitario requiere un uso constante de datos científicos para orientar las intervenciones. Las decisiones en cuestiones de salud logran ser más eficientes en la medida en que estén fundamentadas en información basada en conocimientos científicos aplicados en contextos específicos. En consecuencia, para lograr una mayor equidad en el campo de la salud es indispensable que la 
información científica en diferentes medios, formatos, paquetes y lenguajes sirva de base para tomar decisiones en torno a políticas, planificación, gestión, investigación, educación, servicios y atención de la salud. La formación de la Biblioteca Virtual en Equidad, Salud y Desarrollo Humano permitirá crear y consolidar escenarios en que las decisiones en torno a la salud se basen progresivamente en información cientificotécnica.

\section{CONTEXTO Y BASE DE LA ESTRATEGIA PARA PONER EN MARCHA LA BIBLIOTECA VIRTUAL EN EQUIDAD, SALUD Y DESARROLLO HUMANO}

\section{El contexto}

En la BVS, la confluencia de productores, intermediarios y usuarios de información se traduce, en la práctica, en una red dinámica de fuentes de información que son creadas y manejadas en conjunto y de un modo descentralizado, con controles de calidad explícitos. Es muy escaso el conocimiento sobre el comportamiento de los usuarios y productores, de sus hábitos de uso y generación de información, así como de los entornos donde usan esa información. Pero en varios eventos internacionales, los especialistas han reconocido que el entorno informativo de los usuarios y generadores de información se ha enriquecido gracias a la aparición de una oferta de nuevos productos informativos. Por ejemplo, la CEPAL reconoce como una de las principales características del mercado de la información que las personas que toman decisiones tienden a necesitar cada vez más información mejor elaborada. Asimismo, el financiamiento proveniente de la cooperación internacional tiende a disminuir o cambiar en su estructura y probablemente su destino.

Lo anterior nos lleva a pensar que los procesos de información requieren grados progresivamente mayores de creatividad y liderazgo para captar e identificar las necesidades de esos usuarios y generadores y de quienes toman decisiones, así como sus metas a largo plazo y sus intereses operacionales en el corto y el mediano plazo. Esto significa comprender los ejes temáticos en los que están centrados sus discusiones y sus problemas, y su necesidad de estar plenamente informados sobre las áreas de su interés.

En diversas esferas se ha planteado de manera reiterada la necesidad de revisar las estrategias de comunicación, así como los contenidos de los mensajes y los diferentes ambientes donde se usa la información. También se ha expuesto la necesidad de hacer un examen que vaya más allá de las ventajas tecnológicas de la oferta y que lleve al estudio de una política de comunicaciones y de diseminación de información en las instituciones.

Cabe resaltar que la "exclusión digital" no se supera solamente proporcionando acceso a las fuentes de información internacionales que hay en la Internet. La "inserción digital" significa contar con la capacidad local para manejar fuentes de información basadas en contextos locales y conectadas a los flujos internacionales de la corriente principal. ${ }^{4}$

En el contexto enunciado, diversas instituciones de la Región y la OPS, en coordinación con BIREME, se han propuesto desarrollar una estrategia para diseminar información cientificotécnica sobre la equidad, la salud y el desarrollo humano, tomando como modelo la BVS. Para este propósito se llevó a cabo en La Habana, Cuba, en abril de 2001 la Reunión para el Desarrollo de la Biblioteca Virtual en Equidad, Salud y Desarrollo Humano, donde un Comité Consultivo ${ }^{5}$ discutió distintos aspectos de su creación. Posteriormente se formularon recomendaciones en la reunión sobre Estrategias para la Utilización de Información Científica en la Toma de Decisiones en pro de la Equidad en Salud, celebrada en Cancún en junio de 2001. ${ }^{6}$ (Véase Jun y Panisset en esta edición).

\section{La estrategia}

La estrategia se basa en tres conceptos centrales para la salud pública: a) la cooperación, b) la participación de diversos actores sociales y c) la pluralidad. Sus objetivos son:

- procurar que información de calidad sea accesible, aprovechando las ventajas de la Internet, para quienes deben tomar decisiones, a fin de que sirva para mejorar la salud y el bienestar de toda la población, lograr mayor equidad en materia de salud y mejorar el aporte del sector sanitario al desarrollo humano.

- contribuir a mejorar la capacidad de cada país de la Región para elaborar políticas públicas dirigidas a reducir las inequidades en salud y a enfocar la atención en la pobreza, la gobernabilidad,

\footnotetext{
4 Anales del Congreso Regional de Información en Ciencias de la Salud (CRICS V), La Habana, Cuba, abril de 2001.

5 Consulte los documentos de la Reunión en http://www.paho.org/ Spanish/HDP/HDD/bvscuba.htm

6 Consulte los documentos de la reunión en http://www.paho.org/ English/HDP/HDR/hdr-mex.htm
} 
los distintos grupos étnicos, los derechos humanos, el comercio, la integración y el crecimiento.

Líneas principales de acción. El Comité Consultivo de la Biblioteca Virtual en Equidad, Salud y Desarrollo Humano identificó dos líneas principales de acción, a saber:

- Lograr el uso pleno de la información y de los conocimientos existentes, lo cual implica seleccionar dichos conocimientos de acuerdo con su calidad, pertinencia y utilidad, con los procesos de síntesis y transformación a formatos manejables y fácilmente accesibles, o con la legislación que rige su uso.

- Desarrollar y extender las bases de los conocimientos existentes para lograr nuevos conocimientos que ayuden a mejorar la salud, para lo cual es preciso identificar las necesidades de investigación con miras a invertir en ella y poseer la capacidad para analizar y asegurar los conocimientos de nueva adquisición.

Pluralidad del enfoque, de los usuarios y de los productores. La estrategia se enfoca en la acción de instituciones o grupos que la consideran una vía eficaz para apoyar acciones dirigidas a alcanzar las metas de salud para todos. ${ }^{7}$ El desarrollo de la Biblioteca Virtual en Equidad, Salud y Desarrollo Humano es tarea amplia y compleja. No puede ser conducida por una sola institución o departamento. Más bien, requiere un trabajo mancomunado e intenso. Pero cada institución participante puede desempeñar un papel singular en ese proceso, dando oportunidad a que se desarrollen otros aspectos.

Diferentes tipos de usuarios necesitan distintos tipos de conocimientos $y$, por consiguiente, deben tener acceso a información en diferentes formatos y fuentes. El conocimiento derivado de la Biblioteca Virtual en Equidad, Salud y Desarrollo Humano debe contemplar un amplio espectro de temas identificados por categorías, disciplinas y métodos, teniendo presentes las diferentes perspectivas de las agencias o instituciones participantes. La diversidad es esencial y solo puede lograrse en un amplio radio de acción que garantice una pluralidad de enfoques.

Los usuarios deben ser las personas que utilizan los datos para formular e implementar políticas y para evaluar sus efectos una vez que hayan sido

Alleyne GAO. Information: A Bridge over the Divide. Discurso presentado en el CRICS V, La Habana, Cuba, 24 de abril de 2001. adoptadas. Estos usuarios trabajan en los niveles nacional y local.

Aunque es necesario recopilar más información a fin de elaborar políticas sobre equidad y salud, ya hay una gran cantidad de datos que deberán ser utilizados para apoyar las necesidades de los usuarios.

\section{El papel del Comité Consultivo de la Biblioteca Virtual en Equidad, Salud y Desarrollo Humano}

El Comité tiene carácter consultivo y coordina el trabajo conjunto; define los criterios de calidad que deben cumplir las fuentes de información; define prioridades; fomenta la división de responsabilidades en la operación cooperativa de las fuentes de información, y controla y evalúa cada una de estas fuentes y el desempeño de la Biblioteca Virtual en Equidad, Salud y Desarrollo Humano como un todo.

Teniendo en cuenta la amplitud del tema de la equidad, salud y desarrollo humano, habrá que darle prioridad al desarrollo de la correspondiente Biblioteca Virtual. Las prioridades estarán sujetas a varios factores, incluidas las necesidades de las personas y grupos, las agendas políticas y las iniciativas y políticas gubernamentales. El Comité Consultivo puede proporcionar algunos criterios para determinar qué temas se abordarán y cómo medir su impacto. Algunas posibilidades son el tema de la "abogacía"; de la medición de las inequidades (métodos usados para medirlas y los indicadores de equidad); la equidad en las condiciones de vida; la equidad en el estado de salud y la equidad en el acceso a los servicios de salud y en su utilización.

\section{La Biblioteca Virtual en Equidad, Salud y Desarrollo Humano y la práctica}

A pesar de los desarrollos tecnológicos que se han logrado en las ciencias de la computación y la comunicación, no todos aquellos que formulan políticas tienen acceso a fuentes que funcionan en el entorno de la Internet. Es importante, entonces, tener el respaldo de métodos tradicionales, como cintas de video, el contacto personal y hasta documentos impresos en papel. Es indispensable crear alianzas que lleven a la plena utilización de la Biblioteca Virtual en Equidad, Salud y Desarrollo Humano, que busquen la capacitación de sus usuarios mediante guías u otros recursos, y que identifiquen oportunidades para la formación. 
Muchas instituciones crean otros sitios virtuales. Esto no contradice el desarrollo de la Biblioteca Virtual en Equidad, Salud y Desarrollo Humano, pues con las soluciones tecnológicas que existen se pueden realinear los diferentes tipos de bases de datos, no solo para que transfieran información sino para que se comuniquen entre sí.

\section{Contribuciones al debate sobre la equidad y la salud}

A pesar de que el desarrollo de la Biblioteca Virtual en Equidad, Salud y Desarrollo Humano es incipiente-, consideramos que se trata de una oportunidad para apoyar el análisis de políticas tendientes a reducir las inequidades en salud, una especie de oportunidad para mejorar las fuentes de datos existentes, tanto dentro como fuera del sector de la salud, a fin de determinar las desigualdades en este campo. Asimismo, en el área de la "abogacía", la Biblioteca Virtual en Equidad, Salud y Desarrollo Humano deberá convertirse en un instrumento útil para difundir información, insumo clave del trabajo en pro de la equidad en salud.

\section{SYNOPSIS}

\section{The Virtual Library in Equity, Health, and Human Development}

This article attempts to describe the rationale that has led to the development of information sources dealing with equity, health, and human development in countries of Latin America and the Caribbean within the context of the Virtual Health Library (Biblioteca Virtual en Salud, BVS). Such information sources include the scientific literature, databases in printed and electronic format, institutional directories and lists of specialists, lists of events and courses, distance education programs, specialty journals and bulletins, as well as other means of disseminating health information. The pages that follow deal with the development of a Virtual Library in Equity, Health, and Human Development, an effort rooted in the conviction that decision-making and policy geared toward achieving greater equity in health must, of necessity, be based on coherent, well-organized, and readily accessible first-rate scientific information. Information is useless unless it is converted into knowledge that benefits society. The Virtual Library in Equity, Health, and Human Development is a coordinated effort to develop a decentralized regional network of scientific information sources, with strict quality control, from which public officials can draw data and practical examples that can help them set health and development policies geared toward achieving greater equity for all. 